\title{
Query and Service System Based on WebGIS Using in University Choice after the College Entrance Examination
}

\author{
Li-na Guo \\ College of Mining engineering \\ Hebei united university \\ Tangshan, China, 063009 \\ guolina1101@163.com
}

\author{
$\mathrm{Yi} \mathrm{Wu}$ \\ College of Mining engineering \\ Hebei united university \\ Tangshan, China, 063009 \\ 454964268@qq.com
}

\author{
Xiao-gang Wang \\ College of Mining engineering \\ Hebei united university \\ Tangshan, China, 063009 \\ 1422640493@qq.com
}

\begin{abstract}
In view of the current university choosing information query results in intuitive, legibility and comparative difference, space positioning, spatial analysis and visualization expression is not enough, we designed a college entrance examination select query service system based on WEBGIS. The system used ArcGIS for data representation method, used $B / S$ mode, it can realize the query of fractional line of colleges and universities, and the query results can match to the GIS spatial database of map, and it can also realize the query result expression in diversification space expression, thus then it improve the efficiency of information query and selection in the process of choosing; we hope our study may provide some ideas for the GIS product research and development in this direction.
\end{abstract}

Keywords-GIS; college entrance examination; university choice query; GIS products

\section{INTRODUCTION}

In the present and the future for a long period of time, for the chirldren in ordinary families, college entrance examination will still be the most important way to higher education to change their life or destiny. Therefore, examinees and their parents will be very careful of which university and specialty should be chosen, when they face transcript. However, for a long time, most of the university choosing information is recorded on paper. With the development and the popularity of the computer, more and more people begin to make school choice information query web page, university choice web pages...Even though, when there are many results of fuzzy query, the performance results of school choice information that can be gotten on internet is not very intuitive, besides, the legibility and comparative difference is also not good enough. This mainly because most web page information is stored by MIS, and the space positioning, spatial analysis, visualization expression of MIS is good enough, but all the functions mentioned aboved can be implemented by GIS platform.
Based on the reason above, this study is based on ArcGIS technology platform ${ }^{[1]}$, in order to coordinate comprehensive university information to create a service system using in querying university choosing information which is in visual expression, the ordinary information and geographic information are combined to make the universities' location can be gotten with the ordinary text messages, and to display the query results in the form of intuitive figures, tables etc. The system can help high school students choose university effectively, while further help them adapt to university life.

\section{SYSTEM DESIGN}

\section{A. System goals}

The main user is university entrance exam students and their parents, the functions of system is designed to easy to use. After research and analysis, The requirements of users on the system are as follows: (1) Learn more about university admissions over the years; (2) Analyse and compare similar university admissions; (3) Focus on intended university details; (4) learn about university life etc.Therefore, the system is designed to achieve the following functions: score line query, university recommendation, specific university details inquiry, university life service information browsing and interaction.

\section{B. The overall system structure}

The system is based on B / S structure, using Web browser as a client, using Microsoft Visual Studio 2010, Flex Builder3.0 to complete system development, using HTML, MXML for page layout, applying CSS format, and cited part of the files in jQuery to add dynamic effects to web page; JavaScript and ActionScript is chosen as client-side scripting language to achieve system functions, then to relieve pressure on the server. 


\section{Database design}

For any system, the core module is an object-oriented database of different services, database quality will affect the success or failure of the system objectives directly. The system manages spatial data and attribute data by geographic database. The spatial data system is based on "National administrative map", the main layer named "University" is vectorized and symboled by ArcGIS Desktop, and collecting national university-related information in real time. Map publishment is implemente by ArcGIS Server, and the map is sliced before publishment to speed up map loading and reduce the pressure to server ${ }^{[2]}$.

\section{System framework and function module}

The whole system mainly consists of university choosing function module and university life service function module. Among them, the university choosing function module is the core of the system, which can implement functions such as the university basic information query, the university score lines in recent years, and university recommendation according to score. Query results can be shown in form of rich thematic chart ${ }^{[3]}$ (such as pie charts, bar charts, line charts) provided by Flex. The system function framework as shown in Figure 1.

\section{KeY TECHNOLOGY AND APPLICATION}

\section{A. ArcGIS for Server}

ArcGIS is a new generation of GIS software developed by United States Environmental Systems Research Institute, it is one of the most widely used GIS software all over the world. The system is mainly used its ArcGIS for Server software technology, because it can provide a wide range of Web-based GIS services ${ }^{[4]}$, in order to support the achievement of geographic data management, cartography, geography, spatial analysis and editing, and other GIS functionality in a distributed environment.

\section{B. WebGIS technology}

WebGIS is extension and development of geographic information systems ${ }^{[5]}$ working on Internet GIS. WebGIS has the characteristics of traditional GIS and it can implement the retrieve, query, editing and other basic functions of spatial data, at the same time, it also is basis of geographic information dissemination, sharing and collaboration on the internet. WebGIS can make full use of network resources, the foundational processing is arranged for the server to perform, while simple operation for smaller amount of data is done directly by the client.

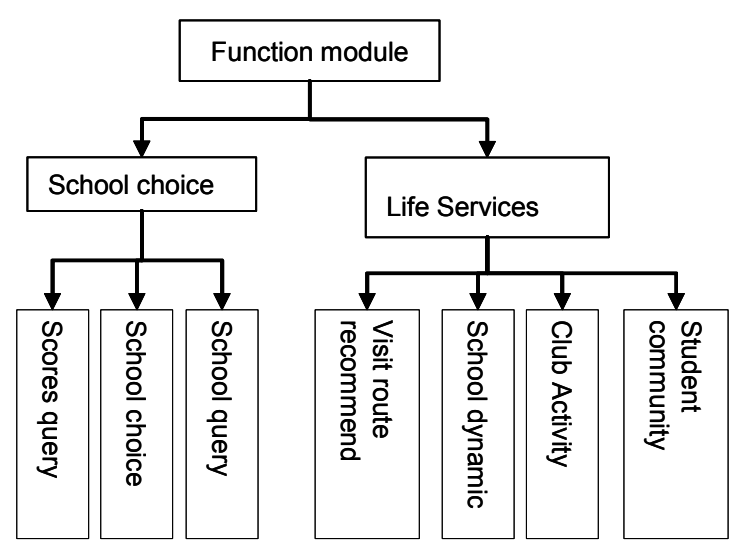

Figure 1 Function framework of the System

\section{THE MAIN FUNCTIONS AND IMPLEMENTATION OF SYSTEM}

The system Web client has three interfaces, arranged in the upper right corner from left to right. They are respectively home interface, information-related interface and map mode interface. Among them, the first two interfaces is designed to implement the "life services" function from vision aspect, university life information browsing aspect and other aspects, map mode interface mainly realize the university choosing function.

\section{A. The implement of home interface and life services function}

Home primarily show some pictures depicted university life and study, guidances about the new life in campus and famous school mottos to users, so users can learn about the upcoming university life from these abstract or figurative information, and make a clear plan of own university life; in information-related interface, users can browse university ranking information independently published by server, keep abreast of the latest data, in order to make the appropriate adjustments of their school choice.This part is mainly used html language for page layout, CSS file to set the style, and cites some files in J-query development package to make development work easier, to gain more vivid interface effects.

\section{B. The implementation of university choosing module}

Click the "map mode" on the top right corner of the home page to enter university choosing module. The mode is divided into two areas, map is in the left area, basic operations of map implement in this area; and the right area is for the university classification and query function, there is "university recommendation" and " secondary function " two function tabs, respectively, to achieve university inquiry and recommendation, surrounding university and scores query.

1) operations of map Use the mouse to achieve the basic operations of map: zoom in or out of the map by moving the mouse wheel, and the drag operation continues as long as 
the left mouse button remains pressed. After initialization, the interface will display a full map, you can see a bubble message box showing the position of each the capital city, open the bubble message box to display a pie chart. The pie chart can demonstrate the quantity and proportion of private school, colleges, undergraduate school clearly and intuitively in each province. When you enlarge the map, detailed university name will display, click the surrounding area of the school, there will pop up a dialog box, which lists the type, address, telephone number and hyperlink URL of school (click URL, you can enter the university homepage in networking status.).

2) university recommendation There are two functions named "university query" and "recommendation for me".

a) University query. Research the database by QueryTask class and attribute value according to the school name entered, then the specific university location will be marked on the map. The elements in search results will marked with InfoSymbol bubble ${ }^{[6]}$, details of elements (such as name, category, address, phone, website, etc, the URL is a hyperlink, users can have a further, more comprehensive understanding after clicking the URl and browsing the website of university) will be displayed in the DetailState state of InfoSymbol. University query function supports fuzzy query. Query result will signed with bubble message box, open the message box fold to view details of universities.

b) Recommendation for me. This function can recommend suitable universities to users according to criteria users selected and score inputed. Users have to select university location, candidate location (different provinces have different score lines), the liberal arts or science, and enter the candidate's score. The system will show the universities that adapt to the conditions selected by users and whose score line less than or similar to the score of candidates.The query results will displayed simultaneously on the map and table, university locations will marked on map in form of bubble message box. Users also can browse the query results in the current table, click the table column names to achieve different orders sorted by the field name; besides, users can pan the map to browse query results, then open the fold of message box marked university position to check the basic information and scores over the years scores of universities to determine their intentions.

3) Secondary function Under the secondary function label, there are three main functions, score line inquiry, surrounding universities inquery and visiting route recommendation.

a) Score line inquiry The data displayed in histogram will refresh immediately when users select province and batch that they want to query, query results will display in a bar graph to provide the criteria arts and science score lines matching the query conditions over the years, including the orange cylinder as score lines of science, and green cylinder as score lines of the arts, when the mouse hovers over the column, the pop-up message box displays the details of the cylinder. Actually, this function did not use the interface provided by Arcgis API for Flex development kits, it simply used the ComoBox control of FLEX to data bounding,
ColomuChart are used to bound score array ${ }^{[7]}$ to display the score over the years visually. Script is writen in ActionScript, the change event of ComoBox will be triggered, then bounding data will change instantly, when the selections in ComoBox change.

b) Surrounding the query This function has used the Geometry service ${ }^{8]}$. Users can click the "site choosing" button on the right side of the interface, then select any point on the map, through the buffer function in the Geometry service, there will show a circumferential extent centered in the point selected, specific options can be set by modifying the buffer parameters.

c) Visiting route recommendation. This module uses NAServer ${ }^{[9]}$ for the route analysis. After selecting "adding site" button, users can click on the main road layer to select the starting and ending point of the route, then the system will calculate the shortest route between starting and ending points and show it, user also can choose to add stagnation point on the shortest route that have been calculated, by clicking "adding barrier" button, the system will calculate another shortest route to avoid this stagnation point.

\section{CONCLUSION AND DISCUSSION}

1) Choosing universities has a great impact on every entrance examinees. The system is designed to provide more comprehensive and intuitive service for examinees, so they can has a good knowledge of universities information. Compared to the current voluntary reporting systems and universities choosing query web pages, the system gathers university basic information, geographic information and score information in one set, adds the map data of universities on the basis of a simple university information query function, the information that examinees need displays on the map directionly to reduce the time to access information. University recommendation function in the system can choose proper universities to examinees according to their scores. On the basis of the implementation of inquiry service, the system adds the release function of university life information, to help the examinees to form a correct understanding of university life, to make the appropriate planning and design for their future campus life. In addition, the system also designed and implemented a series of auxiliary functions, such as surrounding universities inquery and visiting route recommendation, though the two functions can be further enriched and improved.

2) The system achieved a combination of GIS and MIS, maked full use of GIS spatial analysis and graphical representation function, the query results are expressed visually to enhance the visibility, readability of the query results, to play a better assist decision-making role; from the user / bazaar perspective, the system has good value for development, hope to provide some ideas for future GIS product development.

\section{REFERENCES}

[1] Jing Guo. Geographic information Web services [D]. Research and practice of the Chinese people's liberation army information engineering university, 2007. 
[2] Xiaorui Wang, Songfeng Gao,etc. The WebGIS development based on the dynamic cache server and Ajax technology [J]. Journal of surveying and mapping science, 2008 33(5) : 204-205.

[3] [us] tower (Tapper, J.). Flex3 authoritative guide [M]. Beijing: people's posts and telecommunications publishing, 2009.

[4] Qinglei Jia, Qing Wan, Chao Xing. ArcGIS Server development guidelines - based on Flex and. NET [M]. Beijing: science press, 2011.

[5] Jing Wang. The design and implementation of the WebGIS based on Flex [D]. Xi 'an: xi 'an university of science and technology, 2011.

[6] Yu Zhang. WebGIS design and study based on the Flex and ArcGIS Server [D]. Nanjing: nanjing forestry university, 2011.

[7] Xiaopeng Lv. Proficient in flex3.0 - based on actionscript 3 implementation [M]. Beijing: people's posts and telecommunications publishing house, 2008.

[8] Guang Liu, Dashi Tang. Web GIS development - ArcGIS Server and. NET [M]. Beijing: tsinghua university press, 2009.

[9] Jing Zhang. GISWebServices system development research [D]. Shanghai: east China normal university, 2005. 